\title{
Correction to: Tumor location-based classification of surgery-related language impairments in patients with glioma
}

\author{
Shengyu Fang ${ }^{1,2} \cdot$ Yuchao Liang ${ }^{1} \cdot$ Lianwang $^{\mathrm{Li}^{1,2}} \cdot$ Lei Wang $^{2} \cdot \mathrm{Xing} \mathrm{Fan}^{1} \cdot$ Yinyan Wang $^{2} \cdot$ Tao Jiang $^{1,2,3}$ (1)
}

Published online: 12 October 2021

(c) The Author(s), under exclusive licence to Springer Science+Business Media, LLC, part of Springer Nature 2021

Correction to: Journal of Neuro-Oncology

https://doi.org/10.1007/s11060-021-03858-9

In this article Xing Fan and Yinyan Wang should have been indicated as corresponding authors in addition to Tao Jiang.

The original article has been corrected.

Publisher's Note Springer Nature remains neutral with regard to jurisdictional claims in published maps and institutional affiliations.

The original article can be found online at https://doi.org/10.1007/ s11060-021-03858-9.

Xing Fan

xingkongyaoxiang@163.com

$\triangle$ Yinyan Wang

tiantanyinyan@126.com

$\triangle$ Tao Jiang

taojiang1964@foxmail.com

1 Beijing Neurosurgical Institute, Capital Medical University, 119 , the Western Road of the southern 4th Ring Road,

Beijing 100070, China

2 Department of Neurosurgery, Beijing Tiantan Hospital, Capital Medical University, 119, the Western Road of the southern 4th Ring Road, Beijing 100070, China

3 Research Unit of Accurate Diagnosis, Treatment, and Translational Medicine of Brain Tumors Chinese Academy of Medical Sciences, Beijing, China 